\title{
A digital approach for real time high-rate high-resolution radiation measurements
}

\author{
G. Gerardi, L. Abbene* \\ Dipartimento di Fisica e Chimica, Università di Palermo, Viale delle Scienze, Edificio 18, Palermo 90128, Italy
}

\section{A R T I C L E I N F O}

\section{Article history:}

Received 9 May 2014

Received in revised form

3 September 2014

Accepted 15 September 2014

Available online 27 September 2014

Keywords:

Digital pulse processing

Pulse height analysis

Pulse shape analysis

Real time processing

Dead time correction

Energy resolved photon counting

\begin{abstract}
A B S T R A C T
Modern spectrometers are currently developed by using digital pulse processing (DPP) systems, showing several advantages over traditional analog electronics. The aim of this work is to present digital strategies, in a time domain, for the development of real time high-rate high-resolution spectrometers. We propose a digital method, based on the single delay line (SDL) shaping technique, able to perform multi-parameter analysis with high performance even at high photon counting rates. A robust pulse shape and height analysis (PSHA), applied on single isolated time windows of the detector output waveforms, is presented.

The potentialities of the proposed strategy are highlighted through both theoretical and experimental approaches. To strengthen our approach, the implementation of the method on a real-time system together with some experimental results are presented. X-ray spectra measurements with a semiconductor detector are performed both at low and high photon counting rates (up to 1.1 Mcps).
\end{abstract}

(c) 2014 Elsevier B.V. All rights reserved.

\section{Introduction}

Nowadays, spectroscopic systems with high-rate and highresolution capabilities are considered desirable in many physics applications. Generally, "High-rate and high-resolution capabilities" mainly concern the abilities of a system to perform accurate and precise measurement of (i) the input counting rate (ICR) and (ii) the energy spectra even at extreme ICR conditions. At high ICRs, low dead time losses, low variations of energy calibration and a trade-off between energy resolution and throughput must be ensured. Pile-up (tail and peak pile-up) and baseline shifts (mainly due to thermal drifts, poor pole-zero cancellation [1] and AC couplings) are the major drawbacks at high ICR environments and therefore high performance spectrometers must be equipped with both robust pile-up rejectors (PURs) [1] and baseline restorers (BLRs) [1].

A modern spectroscopic system should also perform multiparameter analysis, i.e. provide, besides ICR and energy spectrum, additional experimental parameters for each event, among which: (i) the event arrival time (e.g. for coincidence/anticoincidence measurements), (ii) the pulse shape (e.g. for detector performance enhancements, photon tracking or particle identification) and (iii) the time width of the detected pulses (for dead time corrections).

\footnotetext{
* Corresponding author. Tel.: + 39091 23899081; fax: + 3909123860815.

E-mail address: leonardo.abbene@unipa.it (L. Abbene).
}

Moreover, a fine time evolution of the energy spectrum and counting rates should be provided to analyze variable or transient radiation. Multi-parameter analysis performed at very high fluence rates $\left(>10^{6}\right.$ photons $\mathrm{mm}^{-2} \mathrm{~s}^{-1}$ ) can be very helpful in the development of advanced energy resolved photon counting detectors, recently proposed in diagnostic medicine (computed tomography and mammography), industrial imaging and security screening [2-5].

Currently, the most common high performance spectrometers are developed by using digital pulse processing (DPP) systems [6-19], where the detector output signals (i.e. the output signals from charge sensitive preamplifiers) are directly fed into fast digitizers and then processed by using digital algorithms. DPP systems are typically realized by using two different processing approaches: (i) a personal computer (PC) controls a simple digitizer, receives and records ADC data in files and performs offline processing [14,17-23]; (ii) a PC controls a field programmable gate array (FPGA), equipped with local memory and digitizer; FPGA, wherein pulse processing algorithms are implemented (DPP firmware), receives and processes on-line ADC data, stores results in local memory, gets packets and sends them, when ready, to PC [6-13,15,16,24-26].

Data results are used for quick look processing (for on-line system running control) and are stored in PC files for much deeper off-line analysis [24,25].

As widely recognized, the digital approach gives many benefits against the analog one, among which: (i) possibility to implement 
custom filters and procedures, which are challenging to realize in the analog approach, (ii) stability and reproducibility (insensitivity to pick-up noise as soon as the signals are digitized) and (iii) the possibility to perform multi-parameter analysis for detector performance enhancements and new applications.

Many efforts on the implementation of optimal filtering (trapezoidal, triangular, pseudo-Gaussian, cusp) [27-30] and correction techniques (ballistic deficit, pole-zero cancellation, PUR, baseline recovery) [13,31-34] have been made. However, further improvements are required in the development of high-rate high-resolution spectrometers. To our knowledge, the major limitation of many current DPP systems is represented by their trend to exactly replicate the classical analog pulse processing procedures, showing the same difficulties of the analog systems to mitigate high rate drawbacks. Moreover, due to the potentialities of the DPP approach, more radiation features than ICR and energy spectrum should be provided to the users (e.g. event arrival time, pulse shape, pulse width) to realize non-standard, general purpose radiation detection systems.

In this work we present a DPP method for real time high-rate high-resolution radiation measurements. We propose a method able to perform multi-parameter analysis (event arrival time, pulse shape, pulse height, pulse time width, etc.) even at high ICRs. The pulse detection and the pulse shape and height analysis (PSHA) is carried out by using the classical single delay line (SDL) shaping technique. The PSHA is performed in a robust approach, applied on single isolated time windows of detector output waveforms. First we outline, in a time domain, the typical features of the SDL shaping and its appealing capabilities in pulse detection and in PSHA. Following, the typical high-rate distortions in PSHA, together with possible solutions, will be discussed.

Moreover, to strengthen our approach, the implementation of the method on a real-time system together with some experimental results obtained with a semiconductor detector will be presented.

The aim of this work is to present helpful digital strategies for the development of real time high-rate high-resolution spectrometers. Obviously, these strategies are also suitable for off-line acquisition.

\section{Single delay line (SDL) shaping overview}

In this section, we outline the main features of the classical SDL shaping (also known as delay line clipping) [1,35-37] applied to the typical output waveform from resistive feedback charge sensitive preamplifiers (CSPs), coupled to semiconductor detectors. ${ }^{1}$ SDL shaping is obtained by subtracting from the original pulse its delayed and attenuated fraction. SDL shaping gives short rectangular output pulses with fast rise and fall times. In fact, the falling edge of the pulse is a delayed mirror image of the leading edge. These features make SDL shaping very appealing for timing and PSHA at both low and high counting rates. This classical shaping technique has not been widely used in analog pulse processing, because long-stable delays and stable attenuations are challenging to realize in analog approach. Of course, these difficulties are not present in a digital domain [36,37]. To better explain the properties of SDL shaping, we model a typical single isolated CSP output pulse, with arrival time at $t=0$, as following:

$V_{C S P}(t)=V_{0} \times\left(1-e^{-\left(t / \tau_{L}\right)}\right) \times e^{-\left(t / \tau_{F}\right)}, \quad t \geq 0$,

where $V_{0}$ is an amplitude constant, $\tau_{L}$ is the time constant of the leading edge and $\tau_{F}$ is the time constant of the falling edge $\left(\tau_{F} » \tau_{L}\right)$.

\footnotetext{
${ }^{1}$ Of course, the SDL shaping can be easily applied to reset preamplifier output waveforms, which after high pass filtering (needed to ADC input range interfacing) not require pole-zero cancellation [1].
}

SDL shaping is obtained through the following relation:

$V_{S D L}(t)=V_{C S P}(t)-V_{C S P}\left(t-T_{d}\right) \times e^{-\left(T_{d} / \tau_{F}^{*}\right)}$,

where $T_{d}$ is the delay time and $\tau_{F}^{*}$ characterizes the attenuated fraction. This attenuation, by using the correct $\tau_{F}^{*}$ value (i.e. $\tau_{F}^{*}=\tau_{F}$ ), is able to avoid undesirable undershoots (or overshoots) in the shaped pulses, therefore, working as the classical "pole-zero cancellation technique" [1]. Fig. 1 shows both CSP output pulses and the SDL shaped pulses with different $T_{d}$ values; $T_{p}$ is the peaking time of the CSP output pulse, i.e. the time it takes to reach its full amplitude.

The key features of SDL shaping are reported below:

(i) the time width of each SDL shaped pulse is well defined, in all cases equal to $T_{p}+T_{d}$;

(ii) there is a zero baseline before $(t<0)$ and after $\left(t>T_{p}+T_{d}\right)$ the leading edge of the SDL shaped pulse (using $\tau_{F}^{*}=\tau_{F}$ );

(iii) by using $T_{d}>T_{p}$, the leading edge (pulse height and peaking time) of each CSP output pulse is preserved;

(iv) by using $T_{d}<T_{p}$, the maximum amplitude of the SDL shaped pulse is equal to the CSP value at $t=T_{d}$.

After this general overview on SDL shaping, we will show in the following sections its appealing capabilities for the development of a digital method working at both low and high ICRs.
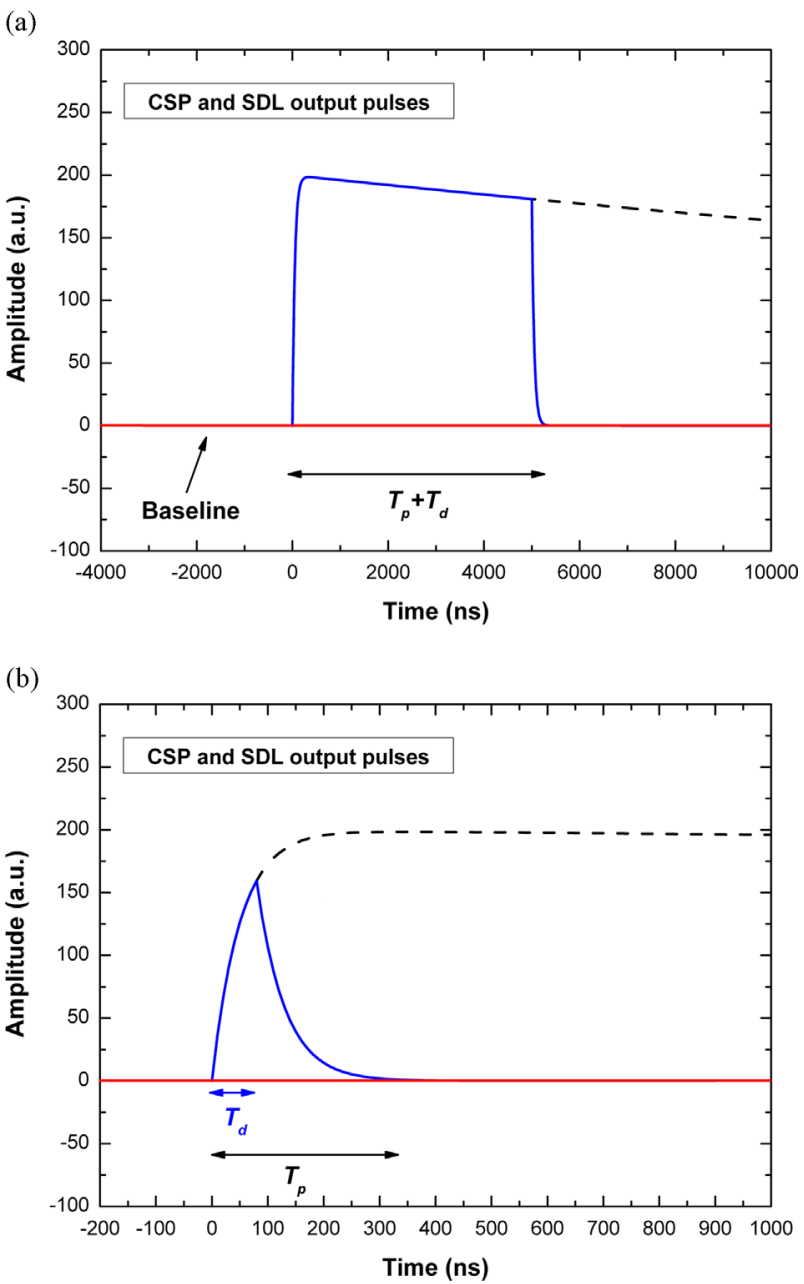

Fig. 1. (color online) (a) Single isolated CSP output pulse (dashed line) with arrival time at $t=0$ and the SDL shaped pulse (solid line) with a delay time $T_{d}>T_{p}$ (peaking time of CSP pulses). Both the leading edge and the baseline of the CSP pulse are preserved. (b) By using a $T_{d}<T_{p}$, the maximum amplitude of the SDL shaped pulse is equal to the CSP value at $t=T_{d}$. 
We will point out the key steps for optimum PSHA using SDL shaping:

(i) pulse detection;

(ii) pulse pile-up rejection (PUR); for each CSP pulse detected, a fixed time window of CSP waveform, centered in the pulse peak position, is inspected; the window is selected for PSHA only if it contains a single isolated pulse; the selected time window of the CSP waveform is termed Snapshot; the width of the time window is termed Snapshot Time (ST); this selection works as a pulse pile-up rejection (PUR); we stress that this PUR only works on CSP peak position times, i.e. it only selects snapshots before any useful operation for PSHA;

(iii) PSHA; the snapshots should be stored in several memories and parallel analyzed to avoid treatment dead times.

\section{Pulse detection using SDL shaping}

The SDL shaping with $T_{d}<T_{p}$ can be very helpful for pulse detection and triggering. To better explain the abilities of SDL shaping on pulse detection, we model a CSP output pulse (with arrival time $t=0$ ), piled-up on the tails of the preceding pulses (tail pile-up), through the following relation:

$V_{C S P}(t)_{\text {TAIL-PILE-UP }}=\left\{\begin{array}{l}V_{\text {TAIL }}(t)=V\left(-t_{0}\right) \cdot e^{-\left(\left(t+t_{0}\right) /\left(\tau_{F}\right)\right)}, \quad-t_{0} \leq t<0 \\ V_{\text {TAIL }}(t)+V_{C S P}(t), \quad t \geq 0\end{array}\right.$

Higher $V\left(-t_{0}\right)$ values model increasing of tail pile-up (i.e. modeling the effects of ICR increasing). The increase of the slope (before and after the leading edge of the CSP pulses, Fig. 2) is not due to changes of the time constant of the falling edge (the time constant only depends on the feedback circuit of CSP) but to the increase of pile-up level.

We also present a model of the CSP output waveform with three pulses (arrival times $t_{1}=0, t_{2}=2 \mu \mathrm{s}$ and $t_{3}=5 \mu \mathrm{s}$ ), characterized by different pulse heights, as following:

$V_{C S P}(t)_{\text {WAVE }}=\left\{\begin{array}{l}V_{\text {TAIL }}(t), \quad-t_{0} \leq t<0 \\ V_{\text {TAIL }}(t)+V_{C S P}\left(t-t_{1}\right)+V_{1} \times V_{C S P}\left(t-t_{2}\right)+V_{2} \times V_{C S P}\left(t-t_{3}\right), t \geq 0\end{array}\right.$

where $V_{1}$ and $V_{2}$ are constants used to obtain different pulse heights. Fig. 2 shows the $V_{C S P}(t)_{W A V E}$ and the SDL shaped waveform with

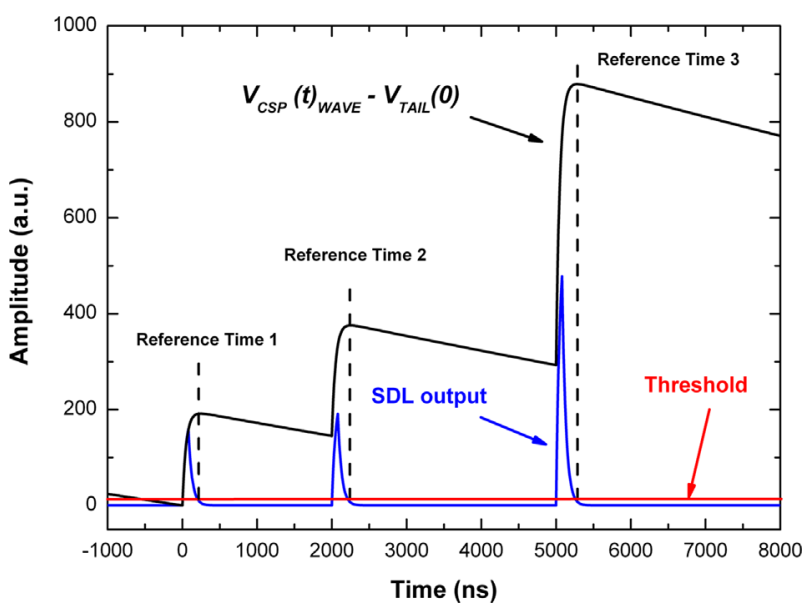

Fig. 2. (color online) CSP output waveform $V_{C S P}(t)_{W A V E}$ with three pulses (arrival times $t_{1}=0, t_{2}=2 \mu \mathrm{s}$ and $t_{3}=5 \mu \mathrm{s}$ ) and the SDL shaped waveform with $T_{d}<T_{p}$. To better highlight the results we reported $V_{C S P}(t)_{\text {WAVE }}-V_{\text {TAIL }}(0)$. The CSP pulse peak detection can be performed through the reference time, defined as the crossing time of the SDL falling edge with the amplitude threshold.

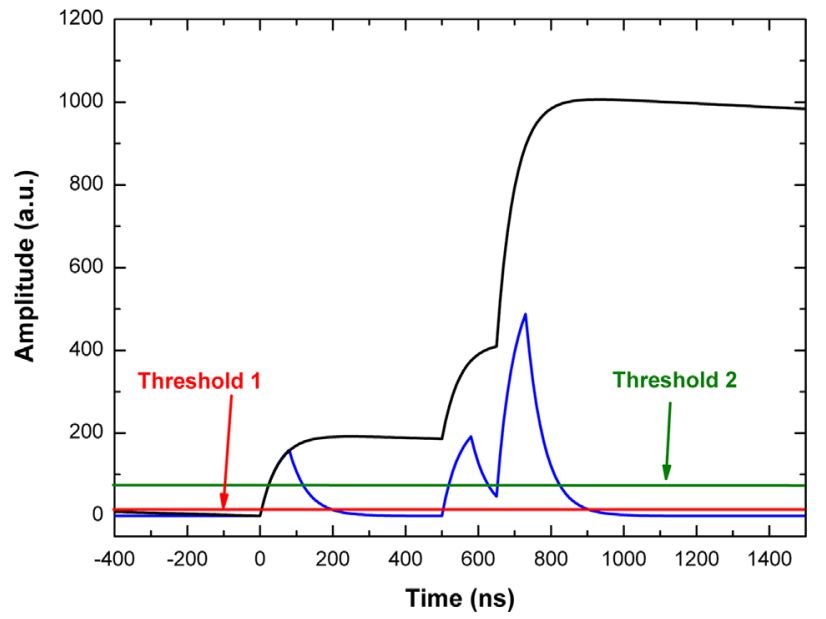

Fig. 3. (color online) CSP output pulses with peak pile-up and the related SDL pulses. The threshold 2 allows to detect the two pulses.

$T_{d}<T_{p}$. To better highlight the results we reported $V_{C S P}(t)_{W A V E^{-}}$ $V_{\text {TAIL }}(0)$. Moreover, Fig. 3 shows a new $V_{C S P}(t)_{\text {WAVE }}-V_{\text {TAIL }}(0)$ with different arrival times $\left(t_{1}=0, t_{2}=0.5 \mu \mathrm{s}\right.$ and $t_{3}=0.65 \mu \mathrm{s}$ and $T_{p}=$ $0.35 \mu \mathrm{s}$ ). Fig. 3 highlights a peak pile-up between two CSP output pulses $\left(t_{3}-t_{2}<T_{p}\right)$.

The key features of SDL shaping pulse detection together with their appealing use are reported below:

(i) a pulse detection is possible by looking for the SDL pulses exceeding an amplitude threshold (leading edge detection); the choice of the threshold depends both on the noise level and on the delay time $T_{d}$ (shorter $T_{d}$ values produce pulses with smaller amplitudes);

(ii) the leading edge detection can be used as time pick-off method to estimate the arrival time of the events (leading edge timing); the effects of time jitter (usually induced by random fluctuations in the pulse amplitude and shape) [1] and amplitude and shape walks (induced by variable pulse amplitudes and shapes) [1] can be mitigated by using the classical amplitude and rise time compensation (ARC) technique [1]. Moreover, further improvements in time pick-off resolution measurements can be obtained through interpolation;

(iii) it is possible to define the time width of a SDL pulse as the elapsed time between the rising and falling edge of the SDL pulse crossing the amplitude threshold; this parameter is very helpful (i) to discriminate event pulses from noise pulses (an event pulse is selected if its time width is greater than a selected time threshold, e.g. $T_{d}$ ), (ii) for dead time estimation;

(iv) the time width of the SDL pulses, as shown in Fig. 3, can be modeled as the classical paralyzable dead time [1];

(v) a CSP pulse peak detection can be performed through the reference time, defined as the crossing time of the SDL falling edge with the amplitude threshold; by using a suitable amplitude threshold and neglecting peak pile-up, it occurs near by the maximum amplitude of the related CSP pulse (Fig. 2);

(vi) as shown in Fig. 3, by using a suitable amplitude threshold (threshold 2) it is possible to recognize peak pile-up pulses. The SDL shaping resolves two consecutive event pulses if the elapsed time between the arrival times of two pulses is greater the time width of a single event pulse;

(vii) moreover, since the maximum pulse time width for a single event pulse can be well defined (e.g. the maximum CSP peaking time $+T_{d}$ ), it can be used to reject pulses with a time width greater than this value, to minimize peak pile-up pulses in the energy spectra; 


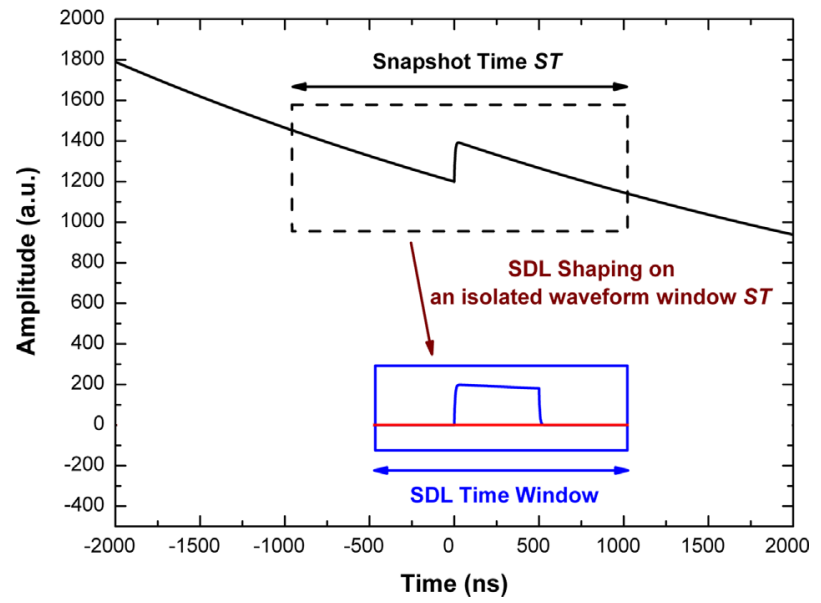

Fig. 4. (color online) Snapshot window (dashed inset) of the CSP output waveform and the SDL shaped pulse (solid inset). Despite pile-up, the SDL output pulse recovers both the leading edge and baseline.

\section{PSHA using SDL shaping}

In this section, we discuss a slow SDL shaping (i.e. with $\mathrm{T}_{\mathrm{d}}>\mathrm{T}_{\mathrm{p}}$ ) applied on the snapshots.

As we discussed in Section 2, by using the correct $\tau_{F}^{*}$ value (i.e. $\tau_{F}^{*}=\tau_{F}$ ) no undesirable undershoots (overshoots) follow and precede the SDL shaped pulses. But using $\tau_{F}^{*} \neq \tau_{F}$ (similar to perform a poor pole-zero cancellation), some distortions follow the SDL shaped pulses. This type of "poor pole-zero cancellation" influences the baseline after the SDL pulse width $\left(t>T_{p}+T_{d}\right)$, producing undesirable overshoots $\left(\tau_{F}^{*}<\tau_{F}\right)$ or undershoots $\left(\tau_{F}^{*}<\tau_{F}\right)$. This result points out that an imperfect attenuation can produce baseline shifts that negatively influence both the accuracy and the precision of the height estimation of the following shaped pulses, especially at high photon counting rates.

The dashed inset in Fig. 4 shows a snapshot $(-S T / 2 \leq \mathrm{t} \leq S T / 2$, with $S T=2 \mu \mathrm{s}$ ) of the CSP output pulse with tail pile-up, centered at reference time $t=0$. A SDL shaping was performed on this isolated snapshot window by using a delay time $T_{d}=500 \mathrm{~ns}(S T / 4)$. The SDL output pulse is within a time window $-S T / 4 \leq \mathrm{t} \leq S T / 2$ (solid inset). Despite tail pile-up, the SDL output pulse recovered both the leading edge and baseline (by using the right decay constant value $\tau_{F}$ and the correct attenuation constant, $\left.e^{-\left(T_{d} / \tau_{F}\right)}\right)$.

However, by using a wrong attenuation constant ${ }^{2}$ and/or imposing DC offsets (simulating the effects due to detector dark current, preamplifier-output offsets and AC coupling) produce, even on isolated SDL pulses, baseline shifts both before the leading edge $\left(t<-T_{p}\right)$ and after the pulse (ST/4 $\left.<t<\mathrm{ST} / 2\right)$, as shown in Fig. 5a. Therefore, despite performing a SDL shaping on isolated pulses, further corrections are needed to obtain an accurate and precise pulse height estimation.

To correct baseline shifts, a baseline recovery can be performed through the following simple procedure: first, the baseline is esti-

\footnotetext{
${ }^{2}$ In real-time FPGA systems, due to using integer arithmetic, wrong attenuations can arise: $V_{C S P}\left(t-T_{d}\right) \cdot e^{-\left(T_{d} / \tau_{F}\right)}=A \cdot e^{-\left(T_{d}\right) / \tau_{F}}$. A way to avoid the use of floating point is to approximate the attenuation, as follows:

$A \cdot e^{-\left(T_{d} / \tau_{F}\right)} \approx A \cdot\left(1-\frac{T_{d}}{\tau_{F}}\right)=\frac{A \cdot \tau_{F}-A \cdot T_{d}}{\tau_{F}}$

If $\tau_{F}$ is an integer power of 2 , the product operation is a simple and fast left shift, and division is a simple and fast right shift. The product $A * T_{d}$ and the subtraction are fast integer operations and easily implemented in a FPGA. If $\tau_{F} \neq 2^{m}$ we must use the $m$ value that produce the nearest value to $\tau_{\mathrm{F}}$. So the attenuation is twofold approximated by the series expansion and by the decay constant approximated by a 2 power value.
}

(a)

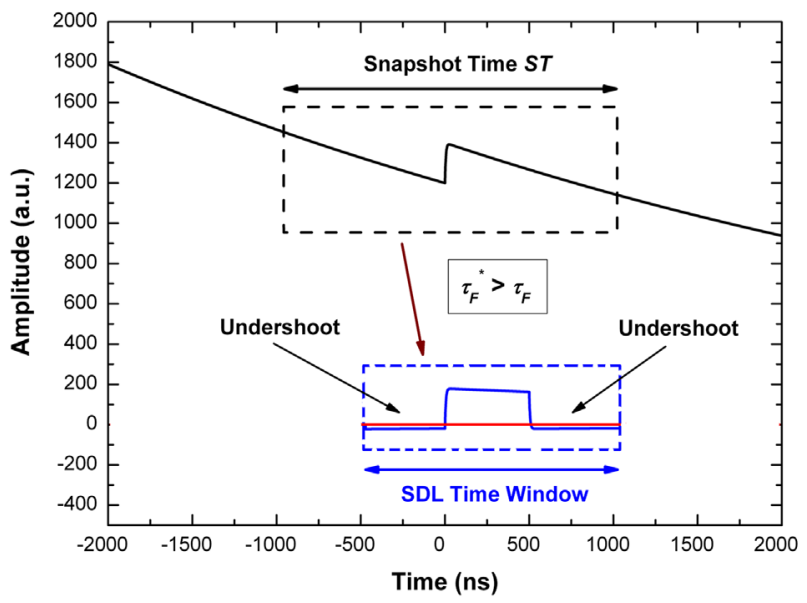

(b)

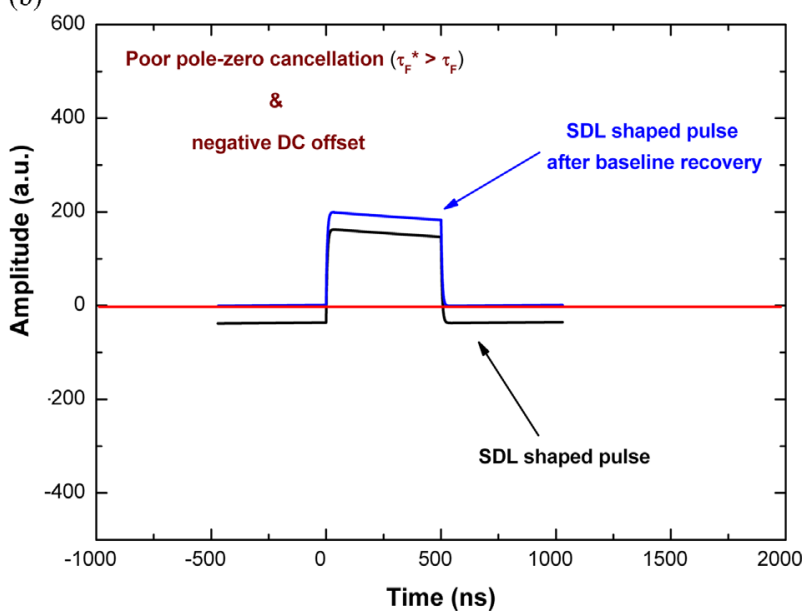

Fig. 5. (color online) (a) Baseline shifts on isolated SDL shaped pulses, due to a poor pole-zero cancellation. Despite performing a SDL shaping on isolated pulses, further corrections are needed to obtain an accurate and precise pulse height estimation. (b) Baseline recovery on isolated SDL shaped pulses characterized by pile-up, poor pole-zero cancellation and a negative DC offset.

mated as the average value of the samples preceding the leading edge of each SDL shaped pulse $\left(-\mathrm{ST} / 4 \leq \mathrm{t}<-\mathrm{T}_{\mathrm{p}}\right)$; then, this baseline value is subtracted from the SDL shaped pulse up to $t=\mathrm{ST} / 2$. Fig. 5b shows the effects of the baseline recovery on a SDL shaped pulse, including both a poor pole-zero cancellation and a negative DC offset.

To better estimate the height of each SDL shaped pulse (after baseline recovery), a compensation of the exponential decay by using a digital deconvolver [37] can be used, followed by the classical trapezoidal shaping for further enhancements in the signalto-noise ratio (Fig. 6). The trapezoidal shaping (flat top included) can be performed within $-\mathrm{Tp} \leq \mathrm{t} \leq \mathrm{ST} / 4$.

\section{Proposed digital approach}

Now, we present our strategy for the development of a highrate high resolution real time system. Based on our previous discussions and on the assumption of a system realized using a PC and digitizer (FPGA controlled), the digital method should be characterized by the following features:

(i) pulse detection, triggering, pulse width measurement and PUR based on a fast SDL shaping (i.e. with $T_{d}<T_{p}$ ); 


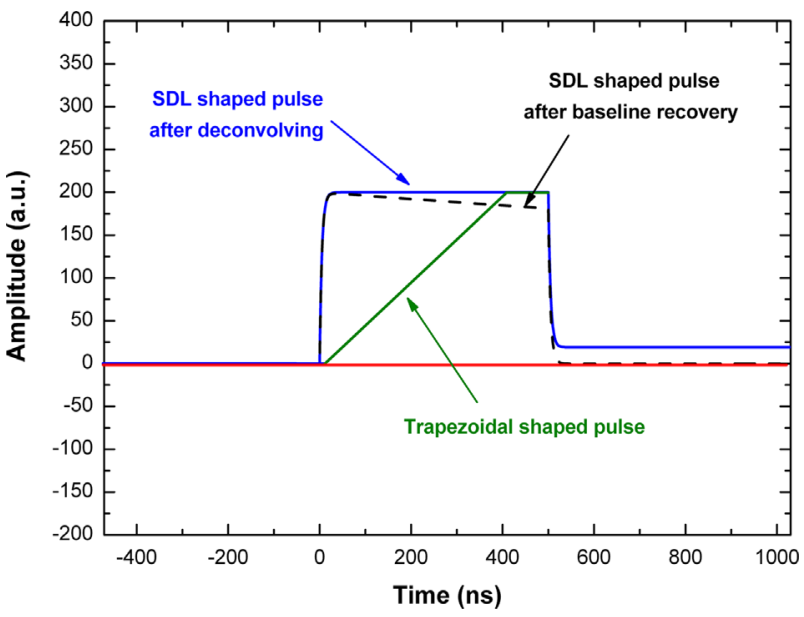

Fig. 6. (color online) The SDL shaped pulse after baseline recovery, after deconvolution and after trapezoidal shaping.

(ii) fast pulse height analysis (PHA) for high throughput measurements by using a SDL shaping with $T_{d}$ very close to $T_{p}$;

(iii) slow (with $T_{d}>T_{p}$ ) pulse shape and height analysis (PSHA) on CSP pulses selected by the PUR for high energy resolution measurements;

(iv) to exploit the ADC input ranges, AC coupling should be also allowed; the baseline recovery can mitigate baseline shifts due to AC coupling;

(v) different working modes to select and provide one or more results from the analysis;

In the following subsections, we will describe the details of our proposed digital approach.

\subsection{Pulse detection, triggering and pulse width measurement}

Once digitized, the CSP output waveform is shaped by using the SDL shaping (i.e. with $T_{d}<T_{p}$ ). Each SDL pulse is detected on exceeding the amplitude threshold (leading edge detection) and the pulse width is greater than the maximum width of a single event pulse. The time when the falling edge of each detected SDL pulse crosses the threshold (reference time) is used to define the center of the snapshot time window in the CSP output waveform. The trigger signal is generated and time-stamped through the ARC timing marker (at the leading edge of the SDL pulses) and, if requested, its amplitude defines the new threshold (ARC threshold) for SDL pulse width estimation. The estimation of the ARC crossing is resolution improved by using a linear interpolation. The width of each SDL pulse is calculated from the difference between the times when the leading and the falling edges cross the ARC threshold or the amplitude threshold. This width is a dead time for the system with a well-defined modeling (paralyzable dead time), allowing the estimation of the true ICR [25]. Moreover, the high-resolution estimation of the trigger allows the measurement of the time intervals between two consecutive events, helpful to validate Poisson statistics and for coincidence/anticoincidence measurements.

\subsection{Fast pulse height analysis (PHA)}

To ensure high-throughput measurements, a PHA can be also performed on the SDL pulses by using a short $T_{d}$ (but with $T_{d}>T_{p}$, to avoid ballistic deficits) and a fast baseline recovery by using the average value of a fixed number of samples preceding the SDL pulse, when no pulses are present. The fast PHA is applied to all detected pulses (PUR is disabled). This fast analysis is foreseen to answer to the requirements (high throughput) of the recent energy resolved photon counting (ERPC) detectors for medical applications, working at very high photon fluence rates $\left(>10^{6}\right.$ photons $\left.\mathrm{mm}^{-2} \mathrm{~s}^{-1}\right)$ [2-5].

\subsection{Pulse shape and height analysis (PSHA)}

To perform the PSHA on single isolated CSP pulses, a pile-up rejection (PUR) is required. The reference time $t_{0}$, (i.e. the time when the falling edge of the SDL pulses crosses the threshold) will detect a single, pile-up free, even if there are no other reference times within the ST time window $\left(-\mathrm{ST} / 2<\mathrm{t}-\mathrm{t}_{0}<\mathrm{ST} / 2\right)$. Once extracted, the snapshot waveform is shaped by using a slow SDL operation, characterized by a longer delay time than the fast one (we used a $T_{d}=S T / 4$, as example). The slow SDL shaping output is used to perform: (i) a baseline recovery, by evaluating the mean value of the samples preceding the leading edge of the shaped pulses (as discussed in section 4), (ii) a pulse shape analysis, by evaluating the maximum amplitude and the rise time of the shaped pulses and (iii) the height analysis of the shaped pulses. The maximum amplitude of the shaped pulses is obtained after compensation of the exponential decay by using the digital deconvolver (Section 4). The pulse height (event energy) estimation is performed by applying an optimized (ST self-adaptive) lowpass filter (e.g. trapezoidal filter) to all the samples of each shaped and deconvolved pulse. Energy resolution strongly depends on ST values; as the shaping time of classic analog systems, long ST values give better energy resolution. The minimum ST value should be equal to $4 * T_{p}$.

The shape analysis consists in the measurement of the peaking time of the shaped pulses. The rise time of the pulses, i.e. the interval between the times at which the shaped pulse reaches $10 \%$ and $90 \%$ of its maximum amplitude, is first evaluated. The times, corresponding to the fractions (10\% and $90 \%$ ) of the pulse height, are obtained through a linear interpolation. The method estimates the peaking time equal to 2.27 times the rise time (i.e. about five times the time constant). The shape analysis is helpful for detector performance enhancements. For example, the correlation between the height and the peaking time of the pulses can be used, to minimize both incomplete charge collection and peak pile-up effects (i.e. overlapped CSP pulses within the peaking time). Since, the peak pile-up pulses are characterized by a longer peaking time and a higher height than the single event pulse, it is possible to reduce their effects in the measured spectra by analyzing both the peaking time and the height (energy) distribution of the pulses [22-25].

\subsection{Data outputs and working modes}

The system, composed of a PC and a digitizer (FPGA controlled), should be able to allow diagnostic tests for optimum parameter setting and return experimental properties of the detected radiation. Therefore, the following data outputs, listed as diagnostic and scientific working modes, must be provided to the users.

\subsubsection{Diagnostic working modes}

(I) CSP output waveform (oscilloscope mode), helpful to check (i) the polarity of the waveform and (ii) ADC saturation;

(II) fast SDL output waveform (oscilloscope mode), helpful to verify (i) the right choice of the decay time constant of the CPS pulses (i.e. if the samples between shaped pulse have an average value as near to zero as possible) and (ii) to set the amplitude threshold;

(III) a sequence of snapshots, helpful to verify the (i) ST value, (ii) if the $S T$ time windows are centered on the pulse peak position, (iii) the PUR performance, i.e. if no other pulses are present in 
the ST time windows, with exception of a possible incomplete leading edge of a next pulse;

(IV) a sequence of time windows, showing the leading edge waveform (i.e. the leading edge of the slow SDL pulses after base line recovery and deconvolution). This output can be used to verify (i) the baseline recovery, (ii) the right value of the CPS decay time (in this case, the samples following each peak are randomly distributed about a constant value), (iii) the right values of the $10 \%$ and the $90 \%$ leading edge thresholds for rise time estimation.

\subsubsection{Scientific working modes}

(I) A sequence of the following three results for each detected event: (i) the arrival time stamp, (ii) the fast SDL pulse height and (iii) the pulse width. These outputs are useful to obtain the time-interval distribution of the detected events, coincidence/anticoincidence measurements, energy spectra with very high throughput, multiscaling acquisitions and the estimation of paralyzable detection dead time from the average of the SDL pulse widths (after peak pile-up correction);

(II) a sequence of the following three results for each detected event: (i) the arrival time stamp, (ii) energy and (iii) peaking time. These data are helpful to obtain energy spectra with high resolution but with lower throughput and to perform multi-parameter analysis, e.g. using the correlation between energy and peaking time. If timing is not required, it is possible to reduce the transmission bandwidth by only providing energy and peaking time values.

Obviously, not all the previous working modes must be carried out simultaneously. For example, diagnostic working modes I to IV can be used for different optimum parameter setting, while scientific working modes, I and II for different radiation investigations. Therefore, only one input/output serial communication channel can be foreseen. Problems can arise from the communication bandwidth. Scientific working mode I, which is characterized by very high throughput at high ICRs, requires more bandwidth than the other one. Additionally, the problem is more serious if the communication channel is used for several detection systems.

Further problems arise from the transmission mode format. To avoid transmission overhead, the event data can be stored in a formatted buffer and transmitted when the buffer is full. To avoid data loss, two buffers, at least, are needed: while a buffer is used for data packing the other one can be transmitted. To optimize the buffer transmission and data retrieval, the buffer length should be fixed, so that the same maximum number of data bytes or packets, must be stored in each buffer.

Whichever working mode is used, each formatted data must be tied to some housekeeping data, such as start time of the packed data acquisition, total value of dead time, total number of fast detected pulses, total number of analyzed events, total number of pile-up events, total number of peak pile-up events. The setting parameters of all working modes must be correctly defined because they are used by the housekeeping data system. These data, continuously updated, can be used for detection and restoration of any transmission dead time losses. Moreover, from housekeeping data, the user can easily obtain, with high time resolution, the count rates and investigate variable or transient radiation, multi-scaling, etc.; from scientific data, a very fine time evolution of the energy spectra and loss counting corrections can be performed.

\section{Implementation of a real-time system}

We present a DPP system for real time radiation measurements, based on our proposed method.
We used a commercial digitizer (DT5724, CAEN S.p.A., Italy), housing four high speed ADCs (16 bit,100 MS/s), four buffers of external memory (1 MByte wide each) and four ALTERA Cyclone EP1C20 FPGAs. Each ADC channel, AC coupled, is characterized by one full scale range $( \pm 1.125 \mathrm{~V})$. Using a set of 14 bit from the 16 bit array, we realized three full scale ranges $( \pm 1.125 \mathrm{~V}$, $\pm 0.5625 \mathrm{~V}$ and $\pm 0.2813 \mathrm{~V}$ ). The digital pulse processing is carried out by the FPGA, in which our DPP method is implemented. The channel FPGA packs output data and sends them to another FPGA that collects asynchronously the packets from all 4 channels and transmits them, via USB channel (or via optical link), to a PC. The $\mathrm{PC}$ runs a $\mathrm{C}++$ program able to control all digitizer functions, to acquire packed data, to produce on-line histograms, counting rate display and to store all received information in dedicated files.

All working modes, listed in the previous section, are implemented in the system. Pictures of some modes can be viewed in our previous works $[24,25]$. Below, we present the practical limitations of the working modes:

(i) diagnostic working modes I and II; each sample is 2 bytes wide, the maximum number of samples is 129792 (if the external memory is 8 MByte wide $129792 * 8$ ). An extra working mode has been added to send in chopped mode both CSP and SDL waveforms packing the same total number of samples. The threshold amplitude is a signed value $(+/-8190$ CAD unit). The delay time of the fast SDL is between 20 ns and 310 ns, with a step size of $10 \mathrm{~ns}$;

(ii) diagnostic working modes III and IV; the maximum number of processed events, $\mathrm{Ne}$, is 255 but the number of samples, $\mathrm{Ns}$, must obey the following relation: $N e * N s \leq 129792$;

(iii) scientific working modes I and II; each mode dedicates 8 bytes at each event; the maximum number of events is equal to $129792 / 4=32448$. A third scientific mode, with no timing data, is also implemented ( 4 bytes are required for each event, so the maximum number of events is $129792 / 2=64896)$. Despite the ADC sampling time being equal to $10 \mathrm{~ns}(100 \mathrm{MHz}$ ADC sampling rate), by using a linear interpolation the trigger times of the ARC crossing are characterized by a resolution of $10 / 16 \mathrm{~ns}$ and peaking time values by 2 ns resolution. The user can choose $T_{p}$ values between 20 and $1150 \mathrm{~ns}$ (using step size of $10 \mathrm{~ns}$ ) and ST values between $4 T_{p}$ and $40 \mu \mathrm{s}$ (using step size of $20 \mathrm{~ns}$ );

(iv) the control and acquisition program reads all the suitable parameter values from two files. From the first, it takes all parameters to program each channel FPGA and the transmission FPGA; from the second file, it takes all the parameters necessary to record the received data in files and to make requested quick look analysis.

\section{Experimental results}

To highlight the high-rate high-resolution capabilities of the realtime system, we present some experimental results obtained with a semiconductor detector. We performed X-ray spectra measurements, at both low and high ICRs, with a thin planar CdTe detector [20]. As well known, thin CdTe/CdZnTe detectors (1-2 mm thick) are very appealing for X-ray spectroscopy in the $1-100 \mathrm{keV}$ energy range [40-47]. We used a planar CdTe detector (XR100T-CdTe, S/N 6012, Amptek, USA) with a thickness of $1 \mathrm{~mm}$ (absolute efficiency of 64\% at $100 \mathrm{keV}$ ) and equipped with a resistive-feedback CSP.

Experimental results of the proposed method, implemented on both real time and off-line systems, have been already presented in our previous works [20-25]. At low ICRs ( $<1 \mathrm{kcps}$ ), the performance of the systems are similar to the analog ones. For example, energy resolutions of $0.29 \% \mathrm{FWHM}$ at $662 \mathrm{keV}\left({ }^{137} \mathrm{Cs}\right.$ source) and $0.17 \%$ at 
$1333 \mathrm{keV}\left({ }^{60} \mathrm{Co}\right.$ source) have been obtained with a germanium detector (similar to: $0.32 \%$ FWHM at $662 \mathrm{keV}$ and $0.18 \%$ at $1333 \mathrm{keV}$, by using an analog electronics with a shaping time constant value of $6 \mu \mathrm{s})$ [25]. Moreover, spectroscopic enhancements in the CdTe detector (correction of the distortions due to incomplete charge collection) have been obtained by using pulse shape correction (PSC) techniques [22]: energy resolution of $1.6 \% \mathrm{FWHM}$ at $122 \mathrm{keV}\left({ }^{57} \mathrm{Co}\right.$ source) with no correction and of 0.8\% FWHM after PSC [24]. High rate performance with the CdTe detector have been also showed $[24,25]$ : (i) no energy peak position shifts up to $830 \mathrm{kcps}(22.1 \mathrm{keV}$ photopeak of ${ }^{109} \mathrm{Cd}$ spectrum) and excellent energy resolution of $4.6 \%$ FWHM at $22.1 \mathrm{keV}$ at $830 \mathrm{kcps}$ (energy resolution of 3.0\% FWHM at $22.1 \mathrm{keV}$ at $200 \mathrm{cps})$.

In this work, we measured $\mathrm{W}$-target $\mathrm{X}$-ray spectra at different tube currents (i.e. different ICRs, up to $1.1 \mathrm{Mcps}$ ). As discussed in previous sections, both the width of each fast SDL pulse and the snapshot time ST are dead times for the DPP system and they can be modeled as paralyzable dead times [20,25,38,39]. Therefore, the DPP system is then always able to estimate the ICR through the classical paralyzable equation $[20,25]$, the knowledge of the fast dead time and the measured $\mathrm{OCR}_{\mathrm{FAST}}$ from the fast SDL shaping.

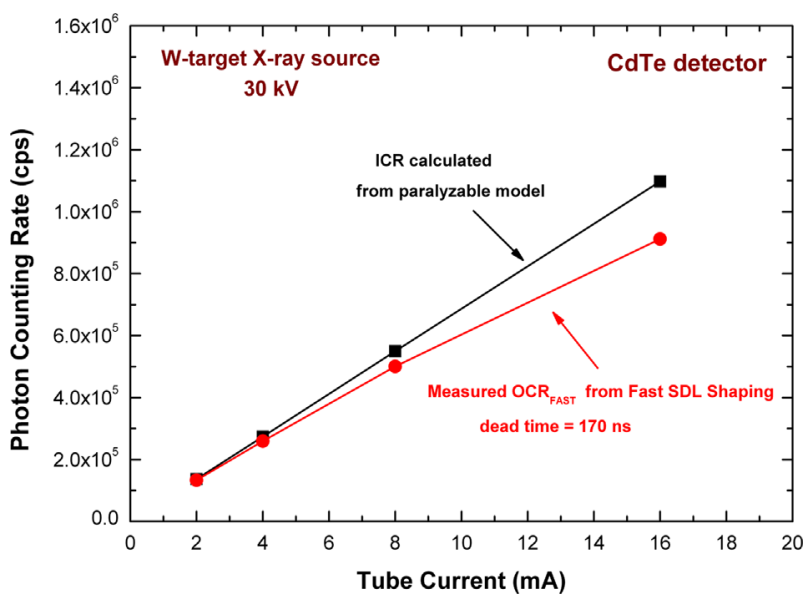

Fig. 7. Measured $O C R_{\mathrm{FAST}}$ from fast SDL shaping versus tube current up to 1.1 Mcps (circles). The ICR calculated from OCR $\mathrm{FAST}_{\text {and }}$ and by using the classical paralyzable equation shows excellent linearity versus the tube current up to $1.1 \mathrm{Mcps}$ (nonlinearity is less than $0.09 \%$ ).

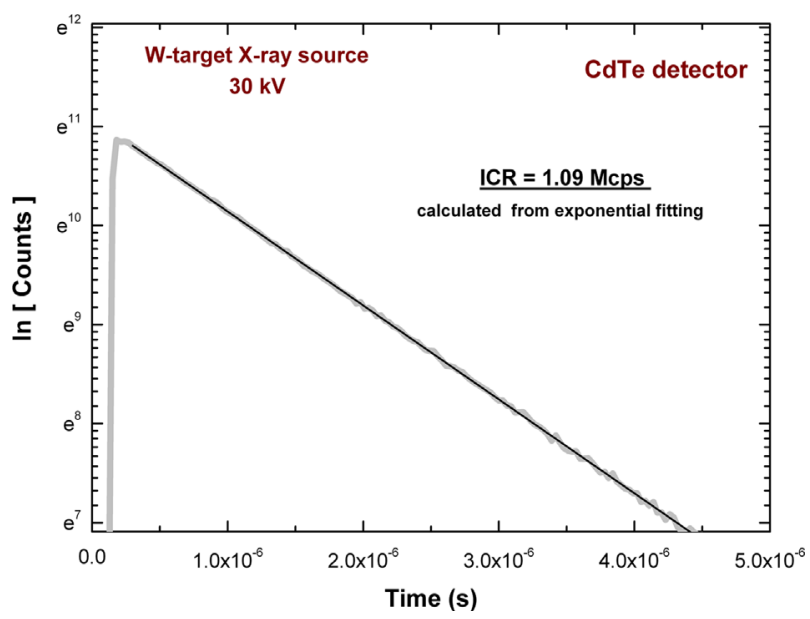

Fig. 8. Measured time-interval distribution of the events detected through the fast SDL shaping. The good agreement between experimental points (gray line) and the exponential best fit function (black line) highlights a Poisson process.
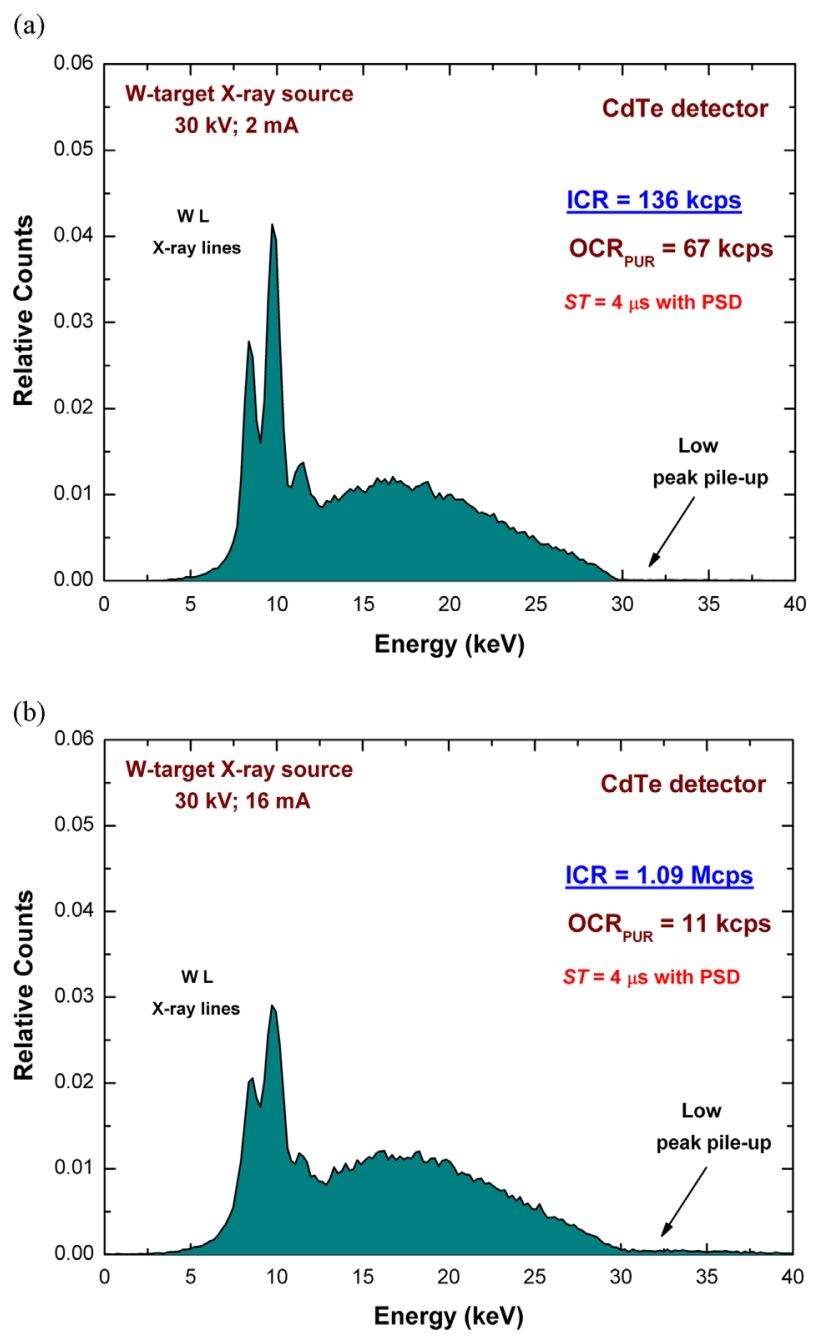

Fig. 9. (color online) Measured W-target X-ray spectrum at ICR of $136 \mathrm{kcps}$ ( $30 \mathrm{kV}$ $2 \mathrm{~mA}$ ). (b) Measured spectrum at very high ICR of $1.09 \mathrm{Mcps}$. Pulse shape discrimination (PSD) technique was used to reduce peak pile-up. The counts were normalized to the total number of the detected events. Energy calibration was performed at low ICR.

Fig. 7 shows both the calculated ICR and the measured OCR FAST from fast SDL shaping (dead time $=170 \mathrm{~ns}$ ) versus the tube current. The calculated ICR versus the tube current shows nonlinearity $<0.09 \%$ up to 1.1 Mcps. We also estimated the ICR by analyzing (by using an exponential best fit) the time-interval distribution of the events detected through the fast SDL channel (Fig. 8). These results highlight that our DPP system is always able to estimate the true rate of the impinging photons through the fast SDL shaping. Moreover, it is possible to use long $S T$ values (low $\mathrm{OCR}_{\mathrm{PUR}}$ ) for optimum pulse height analysis (i.e. for optimum energy resolution) without perturbing the correct estimation of the ICR.

Fig. 9 shows the $\mathrm{W}$-target X-ray spectra measured at moderate ICR of $136 \mathrm{kcps}(30 \mathrm{kV} ; 2 \mathrm{~mA})$ and at very high ICR of 1.09 Mcps (30 kV; $16 \mathrm{~mA})$.

${ }^{241} \mathrm{Am}$ spectra (main energy peak at $59.5 \mathrm{keV}$ ) measured at both low (by choosing the best ST value for optimum energy resolution) and high throughputs (by choosing the minimum $S T$ value for high $\mathrm{OCR}_{\text {PUR }}$ ) are shown in Fig. 10.

We also presented the ${ }^{241} \mathrm{Am}$ spectrum (Fig. 11) measured at very high throughput ( $80 \%$ ) by using the fast PHA (with $T_{d}=200 \mathrm{~ns}$ ). We used this value as the minimum possible value to avoid ballistic deficit ( $\left.T_{p}=190 \mathrm{~ns}\right)$. 
(a)

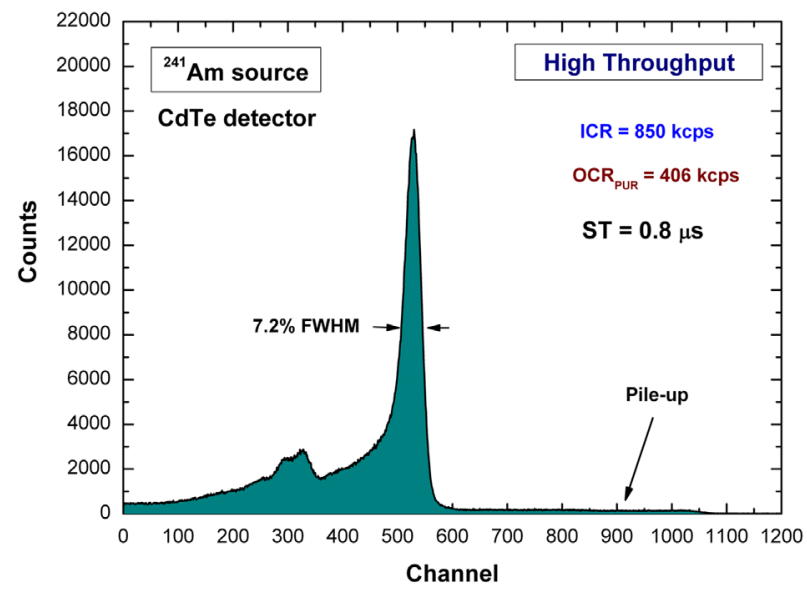

(b)

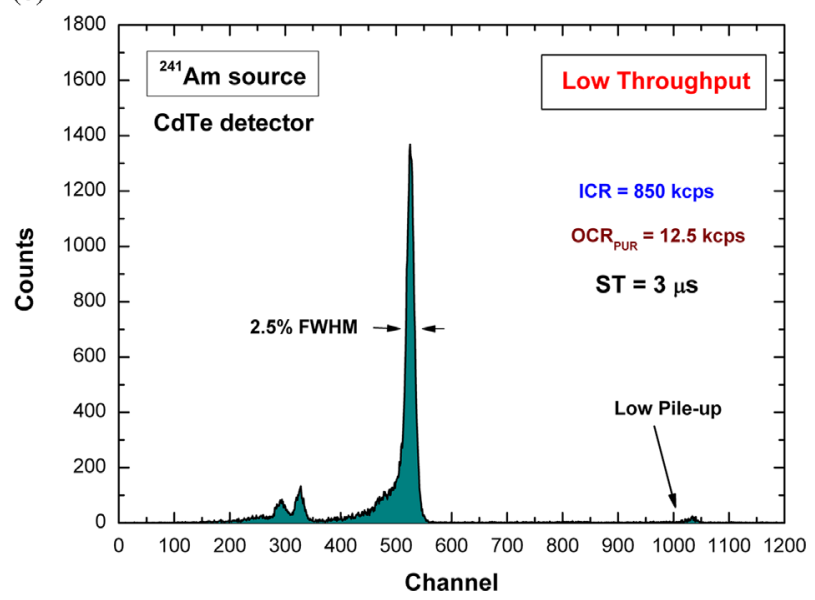

Fig. 10. (color online) ${ }^{241} \mathrm{Am}$ spectra measured at high (a) and low throughput (b). (a) $S T=0.8 \mu$ s is the minimum settable value $\left(4 T_{p}\right)$. (b) Pulse shape discrimination (PSD) technique was also used to reduce peak pile-up.

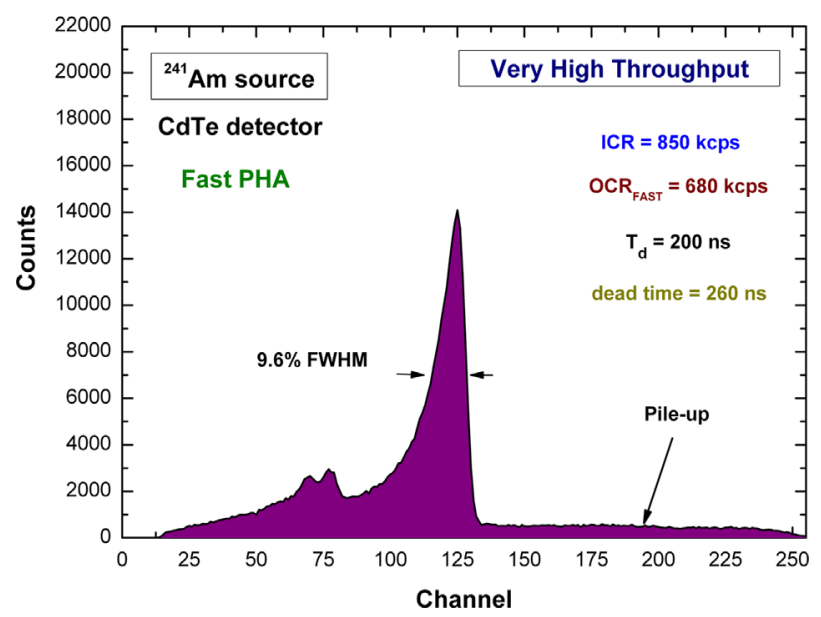

Fig. 11. (color online) ${ }^{241} \mathrm{Am}$ spectrum ( $I C R=850 \mathrm{kcps}$ ) measured at very high throughput ( $80 \%$ ) by using the fast PHA with a $T_{d}=200 \mathrm{~ns}$. The main photopeak $(59.5 \mathrm{keV})$ is characterized by a peak position shift of $6 \%$, if compared with the spectrum measured at moderate ICR (ICR $=14 \mathrm{kcps})$.

\section{Conclusions}

A digital approach for real time high-rate high-resolution radiation measurements was presented in this work. The method, due to both the appealing features of the SDL shaping and the robust PSHA, allows high performance even at high ICRs. Theoretical and experimental investigations highlighted the main capabilities of the proposed method: (i) pulse detection and triggering with high time resolution, (ii) accurate dead time corrections, (iii) energy spectra with high resolution and with no peak position shifts, (iv) multiparameter analysis (event arrival time, pulse time width, ICR, pulse shape, pulse height) through different working modes and (v) the possibility to measure energy spectra at high throughput with dead times close to peaking time of the detector pulses (Fig. 11).

We stress about further key features of the proposed strategy: (i) first, the method is always able to estimate the true ICR, through the fast SDL channel, independently of OCR values in the spectra (one paralyzable dead time without other cascade effects); due to the high time resolution spectra acquisition and the knowledge of ICR, OCRs in the spectra of variable or transient radiation sources are easily corrected (ii) second, there is no treatment dead time by using parallel operations, (iii) third, once the parameters are set for the particular detector, no further settings are required when the input rates changed, contrary to what happens by using conventional pulse processing systems; (iv) fourth, possibility to use, even at high ICRs, long enough $S T$ values (producing low OCR, i.e. low throughputs) for optimum pulse height analysis (i.e. for optimum energy resolution) without perturbing the correct estimation of the ICR.

The method implemented in custom and commercial digitizers is a very attractive tool for both laboratory research and for the development of advanced energy resolved photon counting detectors, recently proposed in diagnostic medicine (computed tomography and mammography), industrial imaging and security screening.

\section{Acknowledgment}

This work was supported by the Italian Ministry for Education, University and Research (MIUR) under PRIN Project No. 2012WM9MEP.

\section{References}

[1] G.F. Knoll, Radiation Detection and Measurement, third ed., Wiley, New York, 2000.

[2] K. Taguchi, et al., Med. Phys. 40 (2013) 100901.

[3] L.F. N.D. Carramate, et al., JINST 6 (2011) C02002.

[4] E. Fredenberg, et al., Nucl. Instr. and Meth. A 613 (2010) 156.

[5] J.S. Iwanczyk, et al., IEEE Trans. Nucl. Sci. 56 (2009) 535.

[6] 〈http://www.ortec-online.com〉.

[7] 〈http://www.canberra.com〉.

[8] 〈http://www.amptek.com〉.

[9] 〈http://www.caentechnologies.com〉.

[10] 〈http://www.xia.com〉.

[11] M. Bolic, V. Drndarevic, Nucl. Instr. and Meth. A 482 (2002) 761.

[12] J.M. Cardoso, et al., Nucl. Instr. and Meth. A 522 (2004) 487.

[13] M. Arnold, et al., IEEE Trans. Nucl. Sci. 53 (2006) 723.

[14] G. Gerardi, et al., Nucl. Instr. and Meth. A 571 (2007) 378.

[15] T. Papp, J.A. Maxwell, Nucl. Instr. and Meth. A 619 (2010) 89.

[16] M. Dambacher, et al., Nucl. Instr. and Meth. A 652 (2011) 445

[17] M. Nakhostin, P. Veeramani, JINST 7 (2012) P06006.

[18] M. Nocente, et al., Rev. Sci. Instr. 81 (2010) $10 D 321$.

[19] M. Nocente, et al., IEEE Trans. Nucl. Sci. 60 (2013) 1408.

[20] L. Abbene, G. Gerardi, F. Principato, S. Del Sordo, R. Ienzi, G. Raso, Med. Phys. 37 (2010) 6147.

[21] L. Abbene, G. Gerardi, S. Del Sordo, G. Raso, Nucl. Instr. and Meth. A 621 (2010) 447.

[22] L. Abbene, G. Gerardi, Nucl. Instr. and Meth. A 654 (2011) 340.

[23] L. Abbene, G. Gerardi, F. Principato, S. Del Sordo, G. Raso, Sensors 12 (2012) 8390.

[24] L. Abbene, et al., Nucl. Instr. and Meth. A 730 (2013) 124.

[25] L. Abbene, et al., JINST 8 (2013) P07019.

[26] P.S. Lee, et al., Rad. Meas 48 (2013) 12.

[27] V.T. Jordanov, et al., Nucl. Instr. and Meth. A 345 (1994) 337.

[28] A. Pullia, Nucl. Instr. and Meth. A 397 (1997) 414

[29] E. Gatti, et al., Nucl. Instr. and Meth. A 523 (2004) 167.

[30] N. Menaa, et al., Nucl. Instr. and Meth. A 652 (2011) 512.

[31] A. Pullia, et al., IEEE Trans. Nucl. Sci. 44 (1997) 331.

[32] A. Pullia, et al., Nucl. Instr. and Meth. A 439 (2000) 378. 
[33] L. Bardelli, et al., Nucl. Instr. and Meth. A 560 (2006) 524.

[34] M. Nakhostin, et al., Rev. Sci. Instr. 81 (2010) 103507.

[35] E. Tanaka, et al., Nucl. Instr. and Meth 158 (1979) 459.

[36] M. Bolic, et al., IEEE Trans. Instr. Meas 59 (2010) 122.

[37] V.T. Jordanov, et al., Nucl. Instr. and Meth. A 353 (1994) 261.

[38] U. Engeland, et al., Phys. Med. Biol. 43 (1998) 2939.

[39] D.F. Yu, et al., Phys. Med. Biol. 45 (2000) 2043.

[40] T. Takahashi, et al., IEEE Trans. Nucl. Sci. 48 (2001) 950.
[41] S. Del Sordo, et al., Sensors 9 (2009) 3491.

[42] L. Abbene, et al., Nucl. Instr. and Meth. A 583 (2007) 324.

[43] A. Owens, et al., Nucl. Instr. and Meth. A 531 (2004) 18.

[44] N. Auricchio, J. Appl. Phys. 110 (2011) 124502.

[45] F. Principato, et al., J. Appl. Phys. 112 (2012) 094506.

[46] L. Abbene, et al., Nucl. Instr. and Meth. A 730 (2013) 135.

[47] A.A. Turturici, et al., Nucl. Instr. and Meth. A 763 (2014) 476. 